\title{
The association between a priori and a posterior dietary patterns with perceived stress in women of childbearing age
}

\author{
Karim Khaled, Orouba Almilaji, Mareike Köppen, Vanora Hundley and Fotini Tsofliou \\ Bournemouth University, Bournemouth, United Kingdom
}

\section{Abstract}

More than half of women in developed countries are overweight or obese. Studies show that $20-25 \%$ of women in the UK enter pregnancy as obese. Healthy diet patterns such as the Mediterranean diet (MD) are linked with lower adiposity among women of childbearing age. However there is evidence that maternal diet in the UK is poor and stress increases the consumption of saturated-fat, sweets, and energy-dense foods. There is limited evidence on dietary patterns and stress among women of childbearing age, therefore this study aimed to investigate the association.

This was an anonymous online survey of female university students. The survey included: socio-demographic characteristics, physical activity, self-reported BMI and waist circumference. Dietary assessment was done via a validated 100 -food item food frequency questionnaire and perceived stress via the Perceived Stress Scale (PSS). The dietary data were compared to the a priori defined Mediterranean Diet Score (MDS) and scored according to eight components (vegetables, legumes, fruit and nuts, cereals, fish, dairy products, meat and meat products, and the ratio of unsaturated to saturated fat). The scores ranged from 0 to 8 with MDS adherence groups being 0-3 low, 4-5 medium, and 6-8 high. Factor analysis was also applied on 11 food components (g/d) (the eight above plus eggs, potatoes, sweets and drinks) to reveal the latent major dietary patterns (DP) in the studied cohort.Negative binomial regression was used to examine the association between stress and the diet patterns, controlling for the socioeconomic factors, physical activity, marital status, BMI, and waist.

One hundred twenty-three students (mean age 27.7 (SD 7.3)) participated. Participants had medium $(\mathrm{n}=49)$ or low MD adherence $(\mathrm{n}=48)$, with fewer participants having high adherence $(\mathrm{n}=26)$. Stress was not significantly associated with MD adherence, but was positively associated with sweets intake $(\mathrm{p}=0.03)$. The scree plot of Factor analysis showed that the number of factors generated by the analysis is three.

Using minimum loading cut-off of 0.4, factor analysis revealed three latent diet patterns; the first (DP-1) contained: cereals, sweets and potatoes (comfort foods), DP-2 consisted: eggs, fish and meat (high protein) and DP-3 consisted of: vegetables, fruits, nuts, and legumes (vegan). Results showed a significant positive association between stress and DP-1 ( $<<0.01)$. No association was found between other diet patterns and any other psychosocial and physical variables.

Future well designed randomised controlled trials are needed to investigate further the relationship between perceived stress and dietary patterns in women of childbearing age.

\section{Conflict of Interest}

There is no conflict of interest 